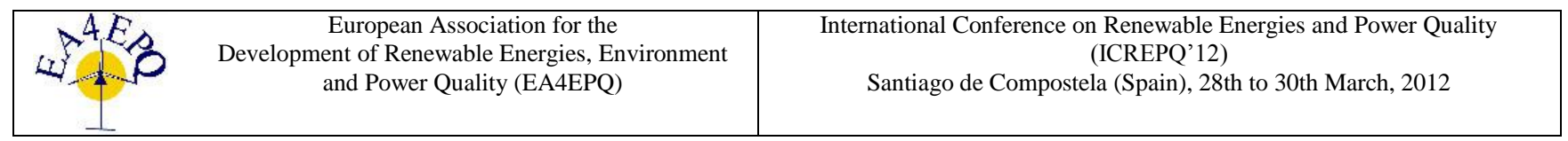

\title{
Characteristics of Twin-Runner Darreus Water-Turbines for Tidal Current Power Generation
}

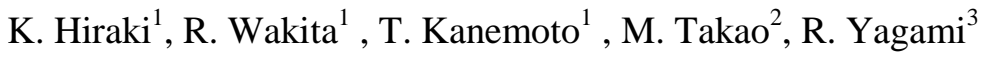 \\ ${ }^{1}$ Department of Mechanical and Control Engineering \\ Kyushu Institute of Technology \\ 1-1 Sensui, Tobata, Kitakyushu, Fukuoka 804-8550 (Japan) \\ Phone/Fax number:+81 93884 3154, e-mail: hiraki@mech.kyutech.ac.jp \\ $2 *$ Matsue College of Technology \\ 14-4 Nishi-ikuma, Matsue, Shimane 690-8518, JAPAN \\ ${ }^{3} *$ Kitakyushu National College of Technology \\ 5-20-1 Shii Kokuraminami, Kitakyushu, Fukuoka \\ 802-0985, JAPAN
}

\begin{abstract}
In order to make the electrical-power generation feasible under the lower tidal-current condition the twin-runner Darreus water-turbine was developed. The counter rotations of twin runners are expected to increase the power output. In order to find out the best distance between the runners the wind-tunnel tests using the subscale model were carried out. At the dimensionless distance of 0.18 the rotational numbers were increased up to 156 percent of those at the single-runner configuration. With this distance the water-channel test was also conducted. It was confirmed that the revolution numbers of the twin runners were increased by 20 percents of those in the single-runner configuration. The increasing effect of speed by a diffuser was investigated. At the stand-alone test of a diffuser in the wind tunnel the velocity of the current passing through the diffuser was accelerated by 1.1 of the free-stream velocity, however, in the water-channel test the subscale Darreus model with the shorter diffuser showed no increasing effect. This was primarily because of the short length of the diffuser to the mouth distance, which was determined from the standpoint of the structural efficiency. The demonstrative power generation test is planned at the Kanmon strait in March, 2012.
\end{abstract}

\section{Key words}

Tidal current, Darreus water turbine, Strait

\section{Introduction}

A series of fatal accidents at Fukushima Daiich Nuclear Power Plants in Japan which was initiated by the tragic earthquakes and tsunami turned the Japanese people's eyes to the cleaner and safer energy sources, that is, the renewable energy. One of the promising renewable energy sources is the ocean, since the Japanese islands are surrounded by the sea. Several ways of utilizing the ocean energy are studied so far, such as ocean current, wave and thermal-difference. Above all, the tidal current power generation is regarded as a most suitable method of power generation for Japan because there are number of straits where the strong tidal current exits.
Considering the tidal-current power generation in a narrow strait, a water turbine with a vertical axis is preferred, since the multiple-stage system is applicable in a depth direction. The simplest but high-performance water-turbine for such purpose is Darrieus-type one with straight-blades. The authors have been developing the power-generating system with Darrieus water-turbine which is suitable for a tidal current in a strait. ${ }^{[1]}$

\section{Twin-Runner Darreus Water Turbine}

In this system a pair of the two-staged Darrieus runners is placed in parallel. These runners rotate counter each other. Both rotations of the runners are transmitted to the doublerotor electric generator. This unique generator equips not only a single rotor but also another rotor instead of a stator as is usual in a conventional electric generator. ${ }^{[2]}$ Twin rotors revolve counter each other, and then the relative revolution numbers in the generator become double. This means that even the lower revolution can produce higher electricity. This system can be applied to the environment where the tidal current is not so strong.

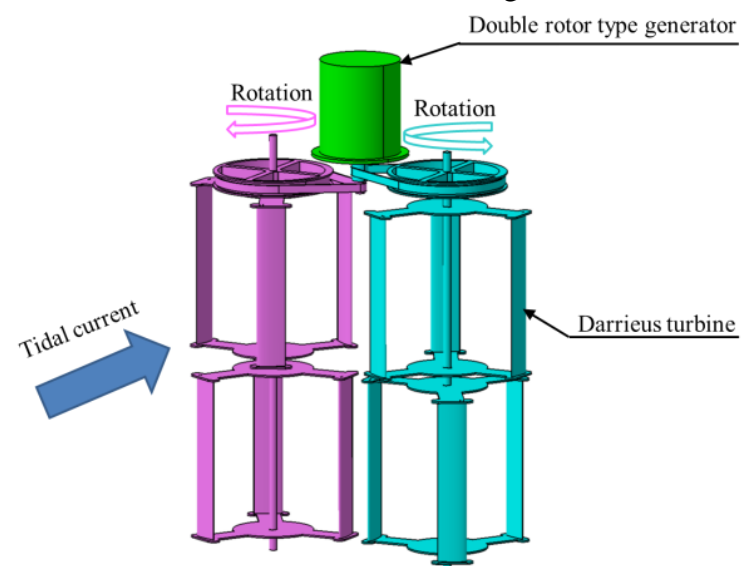

Fig.1 Schematics of twin-runner Darreus water turbine 


\section{Enhancing Effect of Counter-Rotation}

In order to find out the best distance between the two runners both the wind-tunnel and the water-channel tests were carried out. A subscale single-stage model was fabricated for that purpose. The tested model has the runners with a diameter of $160 \mathrm{~mm}$. Each runner consists of the three straight blades of which wing section is NACA0018.

In the wind-tunnel test the wind speed was set to $5.6 \mathrm{~m} / \mathrm{s}$. The gap between the twin runners was varied, and the rotational numbers of the runners under the load-free condition were measured.

In Fig. 2 the variation of the rotational numbers were plotted against the distance between the twin runners. The enhancing effect was observed especially at the rotation type 2 , in which the twin rotors rotate, gathering the flow into the central position. Under such configuration, the enhancing effect was recognized, regardless of the gap between the rotors. At the dimensionless gap of 0.18 , the highest peak was achieved both in the rotation type 1 and 2.

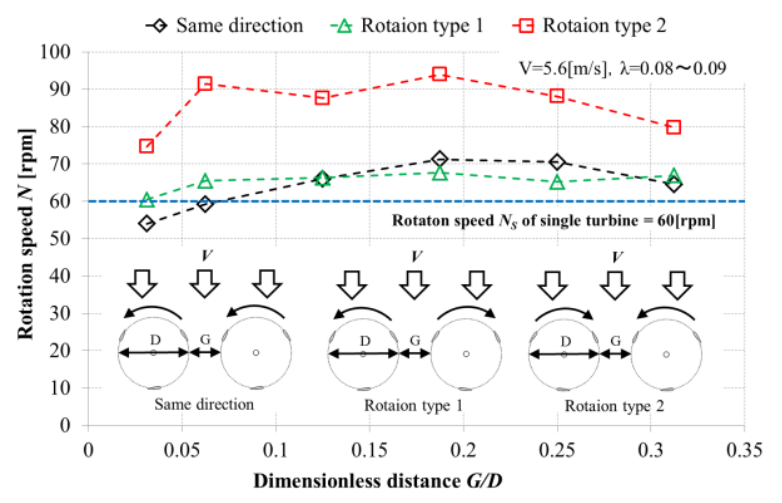

Fig.2 Variation of rotational numbers as a function of a distance between twin runners

Based on the previous discussion, the subscale model with the best distance of the twin runners was tested in a water channel, as was shown in Fig.3. The water velocity in the test channel was $0.83 \mathrm{~m} / \mathrm{s}$. In this test the effect of the rotating direction was investigated. Fig.4 showed the obtained results. It was confirmed that the revolution numbers of the runners in the twin configuration were increased by 20 percents as compared to those in the single-runner configuration, regardless of the rotational directions.

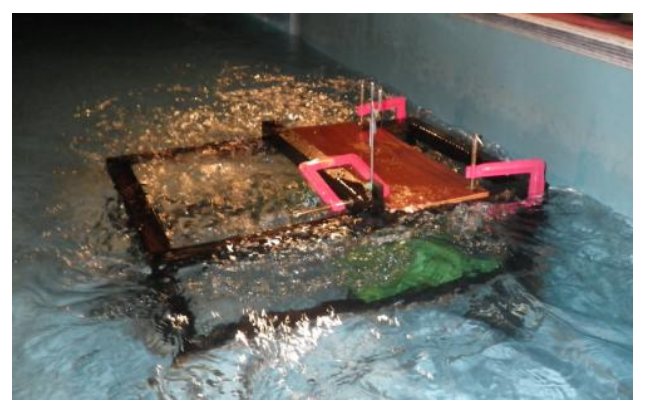

Fig.3 Twin-runner subscale model tested in water channel

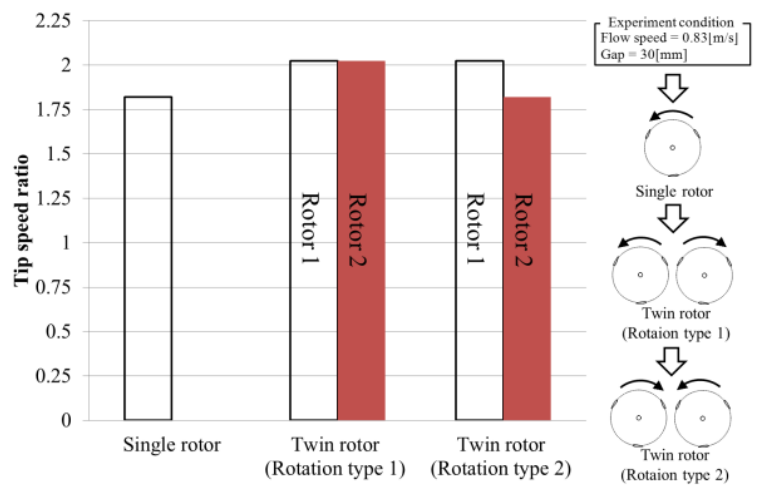

Fig.4 Comparison of tip-speed ratios of twin runners in different directional rotations

\section{Increasing Effect of speed by Diffuser}

In order to investigate the increasing effect of a speed by the attachment of a diffuser, first a standalone test of a diffuser was carried out in a wind tunnel. The test parameter in the test was the expansion angles of the diffuser, as was shown in Fig.5. The velocity of a stream inside of the diffuser was found increased. The peak velocity inside of the diffuser was divided by the freestream velocity and it was plotted against the expansion angle in Fig.6. It was found that the highest velocity was achieved at the expansion angle of 30 degrees. Since the power of the fluid is proportional to the cube of the flow velocity, the increment of the power reaches over 30 percent.

Based on this result, the water channel test was conducted using the subscale twin-runner turbine model with the runner diameter of $160 \mathrm{~mm}$. However, the diffuser length was limited because of the structural efficiency. The water velocity in the test channel was $0.83 \mathrm{~m} / \mathrm{s}$. In this test the match between the placement of a diffuser and the rotational direction was investigated. The relationship of them was illustrated in Fig.7.

The obtained result was presented in the form of the achieved tip-speed ratios of the twin runners in various test conditions. It was found that any configuration with a diffuser did not show better performance than the one without a diffuser. It was primarily due to the short length of the diffuser. In the wind-tunnel test the ratio of the length to the mouth distance of the diffuser was 2.12 , however, it was only 0.18 in the water-channel test. This was insufficient to obtain the enhancing effect of the diffuser.

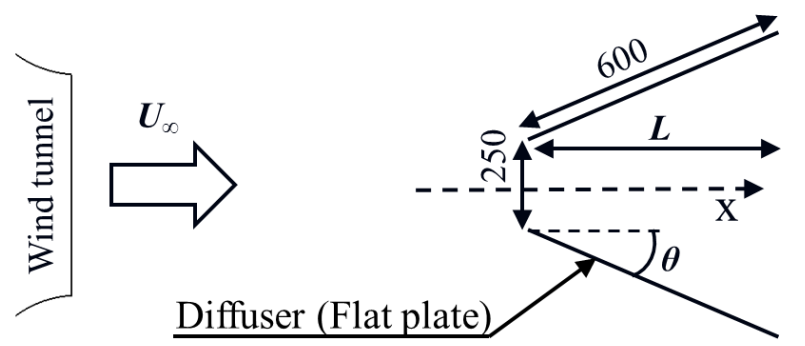

Fig.5 Test configuration of a diffuser in wind tunnel 


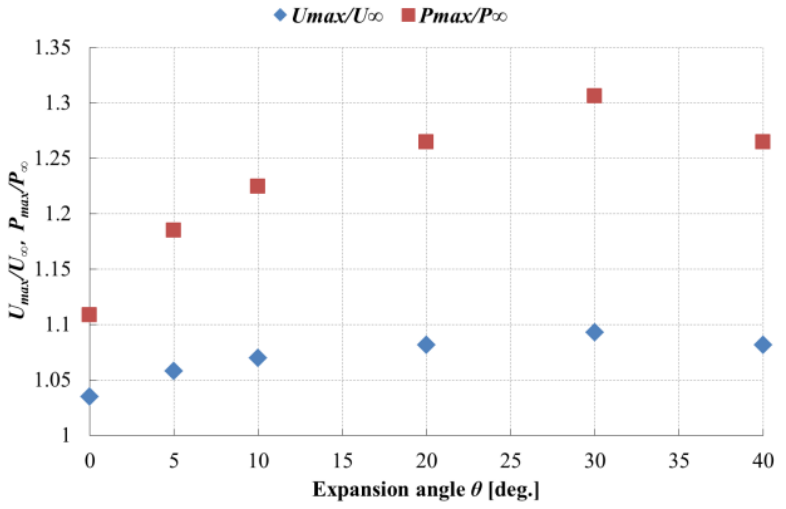

Fig.6 Velocity ratio as a function of expansion angles of diffuser

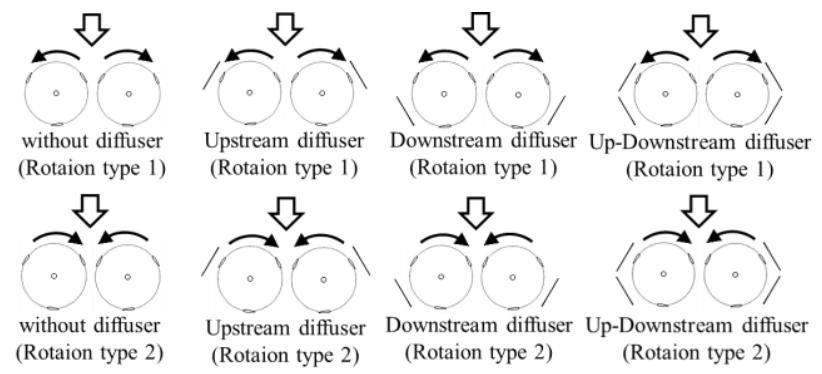

Fig.7 Match of the diffuser attachment and the rotational direction in water-channel test

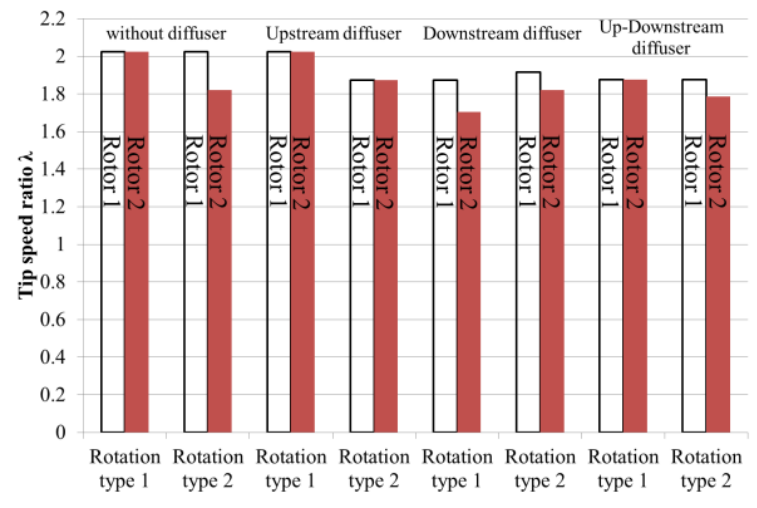

Fig. 8 Tip-speed ratios of twin runners in various configurations of diffuser and rotational direction

\section{Demonstrative Test}

The demonstrative power generation is planned at Kanmon strait, where it is famous for its strong tidal current. The highest tidal current is $4.8 \mathrm{~m} / \mathrm{s}$, however, an area nearby a pier is chosen as the demonstration area, as was shown in Fig.9. The highest tidal current at the place is expected to $1.3 \mathrm{~m} / \mathrm{s}$, which is considered not appropriate to generate electricity. This was due to avoid the conflict with the traffic of ships through the strait. Considering such limitations, thus, the present power-generation system is suitable for the demonstration of its capability under such a low tidal-speed condition. The demonstrative test is slated to get started in March, 2012

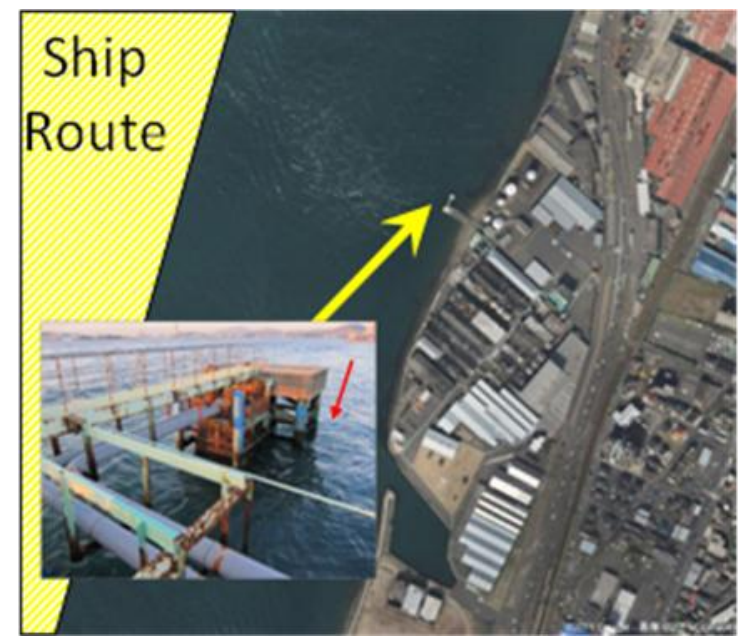

Fig.9 Test location of demonstrative power generation at Kanmon strait

\section{Conclusion}

In order to make the electrical-power generation feasible under the lower tidal-current condition the twin-runner Darreus water-turbine was developed. The counter rotations of twin runners are expected to enhance the power output. In order to find out the best distance between the runners the wind-tunnel tests using the subscale model were carried out. At the dimensionless distance of 0.18 the rotational numbers were increased up to 156 percent of that at the single-runner configuration. With this distance the water-channel test was also conducted. It was confirmed that the revolution numbers of the twin runners were increased by 20 percents of those in the single-runner configuration. The increasing effect of speed by a diffuser was investigated. At the stand-alone test of a diffuser in the wind tunnel the velocity of the current passing through the diffuser was accelerated by 1.1 of the free-stream velocity, however, in the waterchannel test the subscale Darreus model with the shorter diffuser showed any increasing effect. This was primarily because of the short length of the diffuser to the mouth distance, which was determined from the standpoint of the structural efficiency. The demonstrative power generation test is planned at the Kanmon strait in March, 2012.

\section{References}

[1] Koju Hiraki, Ryo Wakita and Masanobu Inoue "Improvement of Straight-bladed Vertical-axis Water Turbine for Tidal Current Power Generation", the 9th (2010) ISOPE Pacific/Asia Offshore Mechanics Symposium.

[2]Kanemoto, T. et al. "Almighty high output intelligent wind turbine generator with tandem wind rotors", (2007),Proceedings of the 5th ASME/JSME Joint Fluid Engineering Conference, CD-ROM FEDSM 2007-37541 